\title{
Análisis de la formación inicial en escuela rural en los Grados de Magisterio: valora- ciones y percepciones del alumnado como agentes implicados
}

\author{
Analysis of initial training on rural schools in Teaching Degrees: evaluations and \\ perceptions of students as agents involved
}

\author{
Silvia Anzano Oto \\ e-mail: sanzano@unizar.es \\ Universidad de Zaragoza. España
}

Sandra Vázquez Toledo

e-mail: svaztol@unizar.es

Universidad de Zaragoza. España

Marta Liesa Orús

e-mail: martali@unizar.es

Universidad de Zaragoza. España

\begin{abstract}
Resumen
La formación para el desempeño laboral en un contexto rural es clave, sin embargo, numerosos estudios han evidenciado un déficit formativo. Analizar la formación que posee un maestro para responder a la especificidad de la escuela rural es esencial para poder adaptar dicha formación a las necesidades de esta. Ante esta situación surge esta investigación de corte cuantitativo que pretende indagar y profundizar en las valoraciones y percepciones de los estudiantes del Grado de Magisterio sobre la formación inicial que han recibido sobre la escuela rural durante sus cuatro años de formación universitaria, así como analizar la posible influencia que tiene el conocimiento y contacto previo con la escuela rural. Los resultados revelan que la formación recibida sobre escuela rural no es suficiente y es excesivamente teórica, los sujetos valoran que no poseen las suficientes competencias, lo que los lleva a percibirse como poco capacitados para afrontar la realidad de su futura labor profesional en este ámbito, principalmente aquellas personas que han vivido alejadas de las zonas rurales. Todos ellos coinciden en que se necesitan mejoras en esta formación, partiendo de la necesidad de vincularla a la realidad práctica. Entre las conclusiones destaca la urgente necesidad de incluir contenidos específicos y con suficiente entidad en la formación inicial de maestros para proporcionarles las herramientas y estrategias necesarias para desarrollar su profesión en el ámbito rural. Respondiendo así a una formación integral del maestro que pueda dar respuesta a las necesidades de la educación del momento y en cada contexto.
\end{abstract}

Palabras clave: escuela rural; formación inicial; maestros; universidad.

\section{Abstract}

Training for work performance in a rural context is key; however, numerous studies have shown a training deficit in rural schools. Analysing the training that a teacher possesses to respond to the specificity of the rural school is essential to be able to adapt such training to the needs of the school. In light of this situation, this quantitative research is aimed at investigating and deepening the assessments and perceptions of students in the Teacher Training Program about the initial training they received in rural schools during their four years of university education, as well as analysing the possible influence of previous knowledge and contact with rural schools. The results reveal that the training received on rural school is not sufficient and excessively theoretical; the subjects' value that they do not possess sufficient skills, which leads them to perceive themselves as not very capable of facing the reality of their future professional work in this field, mainly those people who have lived far from the rural area. All of them agree that improvements are needed in this training, based on the need to link it to practical reality. Among the conclusions, it is important to highlight the urgent need to include content with enough specificity and magnitude in the initial training of teachers to provide them with the tools and strategies necessary for their profession in rural areas. Thus, responding to a comprehensive training of teachers that can meet the needs of education at the time and in each context.

Keywords: rural school; initial teacher education; teachers, university.

Recibido / Received: 20-04-2020

Aceptado / Accepted: 06-11-2020

Publicado en linea / Published online: 22-12-2020

Cómo referenciar este artículo / How to reference this article:

Anzano, S., Vázquez, S., \& Liesa, M. (2021). Análisis de la formación inicial en escuela rural en los Grados de Magisterio: valora-ciones y percepciones del alumnado como agentes implicados. Tendencias Pedagógicas, 37, pp. 43-56. doi: 10.15366/tp2021.37.005 


\section{Introducción}

La escuela rural (en adelante ER) es una realidad en nuestro país - la zona rural en España representa el $85 \%$ de nuestro territorio y el $20 \%$ de nuestra población-, sin embargo, la ruralidad está ensombrecida por un discurso hegemónico urbano-centrista (Vázquez, 2008) que inevitablemente afecta al ámbito educativo.

En este contexto, las escuelas en la zona rural juegan un papel esencial porque garantizan la reducción progresiva de las desigualdades sociales y territoriales, así como la vertebración del territorio. Las escuelas «son un elemento básico en el mantenimiento y trasmisión de las ricas tradiciones socioculturales de la geografía de España» (Recio, 2018, p. 12). Esto supone entender la educación como un servicio público de interés social (Berlanga, 2014).

Si nos sumergimos en la realidad aragonesa, la población censada en 2018 en las zonas rurales en Aragón ha descendido entre los años 2000 y 2018 en 421.321 habitantes (Efe Madrid/Zaragoza, 2019). A pesar de estos datos, durante el curso 2017-2018 Aragón ha tenido 8.496 estudiantes escolarizados en Centros Rurales Agrupados (en adelante CRA). El Ministerio de Educación y Ciencia estableció por primera vez la posibilidad de constituir CRAs en el RD 2731/1986 de 14 de diciembre (BOE del 9 de enero). Estos centros se definen como «aquellos centros que agrupan diferentes localidades dentro de una zona próxima. Es como si fuese un Centro ordinario de una ciudad, pero con "los pasillos muy largos" (en ocasiones de más de cincuenta kilómetros). Son centros con autonomía propia, con un solo Equipo Directivo, con la misma línea educativa, con un Proyecto Educativo de Centro, con un Proyecto Curricular, con un Claustro de profesores y con un Consejo Escolar en el que se vean representadas todas las localidades que pertenecen al CRA» (Mallada, 2004, p. 240).

De los 302.680 estudiantes en edad de escolarización en dicha comunidad autónoma (Ministerio de Educación y Formación Profesional, 2019), el 11,8 \% de la población aragonesa se forma en los 74 CRAs repartidos en las 317 localidades y en 783 unidades (Gracia, 2019). Aragón se constituye como la comunidad con un mayor número de alumnos y alumnas que se forman en ER según los datos proporcionados por el Consejo Escolar del Estado (Ministerio de Educación y Formación Profesional, 2019). Concretamente, se encuentra por detrás de Castilla y León $(21,1 \%)$, Andalucía $(15,3 \%)$ y Cataluña $(14,6 \%)$ y a su vez, se anticipa a Castilla la Mancha $(11,3 \%)$, Cantabria $(0,3 \%)$ y Navarra (0,1\%). En el curso académico 2019-2020, más de 34.100 estudiantes se forman en las aulas rurales, y 85 de estas aulas tienen menos de 10 alumnos (Gracia, 2019). Ya se ha comentado que existen $74 \mathrm{CRAs}$, algunos de estos centros rurales son centros rurales completos, pero en su mayoría tienen aulas unitarias (Gracia, 2019).

La gran mayoría de este alumnado escolarizado en escuela rural tiene que desarrollar su trabajo en un aula unitaria —en adelante AU—, definida por Boix (2004, p. 13) como:

Esa escuela unitaria y/o cíclica que tiene como soporte el medio y la cultura rural, con una estructura pedagógico-didáctica basada en la heterogeneidad y multinivelaridad de grupos de distintas edades, capacidades, competencias curriculares y niveles de escolarización, y con una estructura organizativa y administrativa singular, adaptada a las características y necesidades inherentes al contexto donde se encuentra ubicada.

Y no se puede obviar que este es, probablemente, el primer destino laboral de los maestros y maestras que aprueban una oposición (Avery, 2013). Maestros y maestras con escasa experiencia laboral, generalmente profesorado joven cuyo primer destino es la ER con su aula multigrado. Que experimentan momentos de alegría por iniciar su andadura laboral, pero también de gran incertidumbre por comenzar como maestros de un centro rural, puesto que es una institución con características propias que la hacen diferente a la escuela urbana (Hamodi \& Aragués, 2014). Algunas de estas peculiaridades con las que los maestros se encuentran son: ratios reducidas, alumnado de distintas edades y niveles, recursos limitados o de propia creación, relación más cercana con las familias, implicación de estas en la vida del centro, proyectos innovadores que desarrollar, estrategias, metodologías y evaluaciones muy variadas, etc. (Boix \& Bustos, 2014; Monge, 2019; Abós \& Boix, 2017).

A pesar de la especificidad de la realidad de cada centro, sin lugar a duda, los maestros deben estar formados para este tipo de situaciones o contextos. Sin embargo, la literatura con sus investigaciones de los últimos diez años indica que la formación inicial en ER que reciben los maestros es muy escasa 
y en muchas ocasiones inexistente (Hamodi \& Aragués, 2014; Boix, 2004; Bustos, 2008, 2012; Jiménez, 2009; Moreno, 2000; Hinojo, Raso \& Angustias, 2010).

Ante los datos actualizados de la Comunidad Autónoma de Aragón respecto a su población rural y a la educación que esta debe recibir, así como los indicadores acerca de la formación en esta temática que proporcionan los estudios recientes, se considera oportuno analizar las percepciones y valoraciones de los futuros egresados en magisterio acerca de la formación en escuela rural que reciben en los Grados de Magisterio en la Facultad de Ciencias Humanas y de la Educación de Huesca, en la Universidad de Zaragoza. Asimismo, tiene como fin analizar la posible influencia de sus experiencias previas en la zona o ER (durante su etapa como alumno) en sus perspectivas actuales sobre su profesión en la ER. Pues se considera muy importante recuperar las voces de los estudiantes acerca de sus perspectivas sobre la ER y su formación, con el fin de direccionar mejor el acto educativo universitario (Cifuentes, 2015).

De este modo, tras esta breve introducción acerca de la ER y su estrecha relación con los futuros maestros y maestras, el desarrollo de este artículo tiene como objetivo dar respuesta a las siguientes cuestiones: ¿Los maestros y maestras que actualmente cursan el Grado de Magisterio de Infantil y de Primaria perciben que reciben una formación suficiente para afrontar la realidad y especificidad de la ER? ¿Perciben que las competencias que adquieren durante su formación inicial los capacitan para desempeñar su labor profesional en el ámbito rural? ¿Influye en su perspectiva y valoración el haber tenido experiencias previas, contacto con las zonas rurales o centros rurales?

2. Análisis de la formación inicial sobre escuela rural que recibe el alumnado de Magisterio de la Universidad de Zaragoza

La literatura revisada de los últimos años acerca de la temática manifiesta la escasa oferta formativa para que los estudiantes de Magisterio sean competentes en ER, por esto la realidad educativa actual conlleva a reflexionar y permite argumentar que los futuros maestros necesitan que se les brinden las suficientes oportunidades para pensar cómo planificar su docencia para todo el alumnado, por supuesto, incluyendo aquel de las ER y sus comunidades (Azano \& Stewart, 2015).

Para constatar si este fenómeno se produce en la Universidad de Zaragoza se ha examinado la normativa que regula los requisitos para la verificación de los títulos universitarios oficiales que habilitan para el ejercicio de la profesión de Magisterio en Educación Infantil y en Educación Primaria respectivamente — Orden ECI/3854/2007 y Orden ECI/3857/2007, de 27 de diciembre- De estos documentos se podrían considerar dos objetivos que, de modo implícito, podrían estar vinculados con la ER. Estos tratarían de comprender la función, posibilidades y límites que se pueden encontrar en la educación de la sociedad actual, así como las competencias fundamentales que afectan a los centros educativos de ambas etapas y a los propios maestros. A su vez, enuncia la organización de los colegios y las acciones varias que se requieren para su correcto funcionamiento, habitualmente relativas a la gestión del centro. En este par de objetivos sin duda se debería incluir el ámbito rural, para que así los maestros conozcan sus funciones, la organización y la gestión de la ER.

A su vez, en los estudios universitarios de Magisterio, si se profundiza en la Memoria de Verificación del Grado de Magisterio en Educación Infantil y del Grado de Magisterio en Educación Primaria de la Universidad de Zaragoza, estos objetivos se transforman en Competencias Generales de la titulación y se desarrollan mediante Competencias Transversales y Competencias Específicas. Es en estas últimas —Competencias Transversales — donde se hace referencia a la ER, concretamente en la CT09 para el Grado de Magisterio en Educación Infantil y la CT08 para el Grado de Magisterio en Educación Primaria - Comprender y reflexionar sobre la práctica educativa en entornos rurales-, pero en ningún caso se encuentran Competencias Específicas sobre ER. Y si se analizan las asignaturas del Grado de Educación Infantil y del Grado de Educación Primaria que explícitamente forman en ER, como recogen en su guía docente, únicamente se encuentran tres (Tabla 1) y (Tabla 2).

Tabla 1.

Asignaturas del Grado de Magisterio en Educación Infantil que forman en materia de escuela rural

\begin{tabular}{lcc}
\hline Asignaturas & $\begin{array}{l}\text { Tiene un tema concreto de es- } \\
\text { cuela rural }\end{array}$ & $\begin{array}{l}\text { Tiene bloque de escuela } \\
\text { rural }\end{array}$ \\
\hline La escuela de educación infantil & Sí & Sí \\
\hline
\end{tabular}

Fuente: elaboración propia. 
Tabla 2.

Asignaturas del Grado de Magisterio en Educación Primaria que forman en materia de escuela rural

\begin{tabular}{lcc}
\hline Asignaturas & $\begin{array}{l}\text { Tiene un tema concreto de } \\
\text { escuela rural }\end{array}$ & $\begin{array}{l}\text { Tiene bloque de escuela ru- } \\
\text { ral }\end{array}$ \\
\hline Currículo en contextos diversos & Sí & No \\
La escuela como espacio educativo & Sí & No
\end{tabular}

Fuente: elaboración propia.

Pese a ser un contexto en el que los maestros van a tener que desarrollar su perfil profesional, hasta el curso 2020-2021, se observa que no existen materias que formen a los futuros egresados acerca de este entorno educativo distinto, como es la ER. Si se analizan las asignaturas en las que se imparte ER, son un total de 3 asignaturas. La primera de ellas es obligatoria y correspondiente al tercer curso del Grado en Educación Infantil: «La escuela de educación infantil», en la que existe un tema denominado «La especificidad de la ER»; las otras asignaturas son obligatorias y pertenecen ya al Grado en Educación Primaria, ambas al primer curso, y en cada una de ellas se desarrolla un tema específico de la ER. En «Currículo en contextos diversos» el tema es «La especificidad de la ER. Los procesos de enseñanza-aprendizaje en las aulas multigrado», y a esto se le suma también un resultado de aprendizaje de la misma materia «Comprende la especificidad de la ER y sus necesidades, siendo capaz de diseñar, planificar y evaluar propuestas para la misma». Por último, en «La escuela como espacio educativo» el tema que reseña a la ER es «Estructura y desarrollo legislativo del sistema educativo español: la Educación Primaria y su desarrollo en diferentes contextos (urbano, rural...)».

Cabe destacar que los dos Grados de Magisterio tienen en común una asignatura en la que se hace referencia a la ER. Se trata del Prácticum, en cuyos resultados de aprendizaje se señala «la adquisición de estos resultados de aprendizaje facilita al alumno el conocimiento de la realidad escolar, tanto rural como urbana, en la que va a desempeñar su futuro trabajo». La realización de algunas de las prácticas escolares que se ofertan a lo largo del Grado en una ER hace que el alumnado desarrolle unas determinadas competencias simplemente por estar en entornos singulares, así lo manifiestan Chaparro y Santos (2018a). Es decir, que no se debe ver como una experiencia negativa la obligación de desplazarse a un pueblo fuera de la ciudad para realizar las prácticas escolares en la ER, pues ayuda a desarrollar competencias profesionales (Chaparro y Santos, 2018a) que continuarán ampliando a lo largo de su trayectoria profesional, en la que tampoco olvidarán la renovación de sus saberes y métodos de docencia.

En la misma dirección, determinadas instituciones de algunos países, tras detectar el silencio existente en la formación del profesorado en temas de ruralidad y tomar consciencia de la necesidad real de trabajar la ER, se proponen programas de formación docente destinados a mejorar la formación del maestro rural y su práctica (Masinire, Maringe, \& Nkambule, 2014).

Así, la Ley Orgánica para la Mejora de la Calidad Educativa (LOMCE) añade un nuevo apartado 3 al artículo 9 de la LOE para precisar que «En los programas de cooperación territorial se tendrá en cuenta, como criterio para la distribución territorial de recursos económicos, la singularidad de estos programas en términos orientados a favorecer la igualdad de oportunidades. Se valorará especialmente el fenómeno de la despoblación de un territorio, así como la dispersión geográfica de la población, la insularidad y las necesidades específicas que presenta la escolarización del alumnado de zonas rurales». Y el Consejo Escolar del Estado propone un Plan Nacional de ER.

También desde las comunidades autónomas con competencias en educación se rompe el silencio en relación con la necesidad de formar específicamente para ser maestro en la ER. Como ejemplo, en la Comunidad Autónoma de Aragón, en su Gobierno, se encuentra el amparo legal que las propias Cortes de dicha comunidad han concedido a la ER. Este hecho queda reflejado en diferentes disposiciones, de las que se menciona por su importancia el DECRETO 83/2018 de 8 de mayo del Gobierno de Aragón, por el que se crea el Observatorio de la ER en Aragón, y se regula su organización y funcionamiento (BOA de 17-05-2018). Como apoyo y fruto de una demanda expresa de las Cortes de Aragón, en el curso académico 2020-2021 en la Universidad de Zaragoza se van a incorporar a los Grados de Magisterio algunas asignaturas optativas y semestrales sobre ER que han sido aprobadas 
en las Memorias de Verificación del Grado de Magisterio en Educación Infantil y Primaria. Así, de esta forma se facilita al estudiantado una formación más precisa y concisa de la ER.

Estas nuevas incorporaciones que versan sobre la ER son las asignaturas: «El contexto de la educación en territorios rurales» $\mathrm{y}$ «El proceso de enseñanza en la escuela multigrado», que se presentan como optativas semestrales de 6 ECTS cada una de ellas en el Grado de Magisterio en Educación Infantil. Las mismas asignaturas en torno a la ER se plantean en la Memoria de Verificación de Magisterio en Educación Primaria como materias optativas semestrales de 6 ECTS. En las Memorias de Verificación de los respectivos Grados de Magisterio se plasman los resultados de aprendizaje, los contenidos y las competencias específicas que se detallan en las siguientes tablas, comunes a los dos Grados (Tabla 3 y Tabla 4):

Tabla 3.

Asignatura «El contexto de la educación en territorios rurales»

El contexto de la educación en territorios rurales.

Conoce y utiliza las aportaciones escuela-territorio y viceversa.

Conoce la evolución histórica de la escuela rural y su situación actual a nivel nacional e internacional.

Resultados de apren-

Diseña, desarrolla y evalúa proyectos de centro con implicación del terridizaje torio y la comunidad.

Conoce y maneja estrategias que ayuden a planificar, gestionar y tomar decisiones en la escuela rural.

Analiza y reflexiona sobre el papel de la escuela ubicada en territorios rurales en la sociedad actual.

Conoce y analiza modelos de organización de ER tanto nacionales como internacionales.

Ruralidad, territorio y escuela.

Identidad cultural, sentido de pertenencia y compromiso social.

Identidad cultural, territorio local y territorio global.

Contenidos Proyectos educativos de centro rural multinivel: diseño, desarrollo y evaluación.

Características específicas de la gestión de centros rurales (profesorado, familias, etc.).

La escuela como espacio de convivencia e institución cultural.

Reconocer la diversidad en la práctica educativa en contextos rurales.

Analizar la importancia del papel de la educación para el desarrollo rural y el equilibrio territorial.

Conocer las características y funciones de los principales agentes sociales Competencias especí- y educadores que intervienen en el desarrollo rural.

ficas

Tomar conciencia de la complejidad de la gobernabilidad de los centros educativos ubicados en territorios rurales.

Analizar e interpretar modelos de innovación e investigación (etnográfica)

aplicados a la educación en el territorio rural.

Diseñar un modelo de escuela rural en la sociedad digital.

Fuente: elaboración propia.

Tabla 4. 
El proceso de la enseñanza en la escuela multigrado.

Analiza desde la perspectiva de la escuela rural los elementos que sustentan el proceso de enseñanza-aprendizaje (estilo docente, características del alumnado, del entorno).

Diseña, desarrolla y evalúa proyectos de aula con implicación del territorio

Resultados de aprendizaje

y la comunidad.

Utiliza una pedagogía constructivista respetando los ritmos de aprendizaje

e incidiendo en el uso de metodologías vivas y activas; utiliza el territorio

y la comunidad como recursos.

Plantea la utilización de una evaluación cualitativa.

Utiliza las herramientas y el espacio digital desde la especificidad del territorio rural.

Hacia un currículum contextualizado: diseño y desarrollo curricular en aulas multinivel.

Inclusividad y diversidad.

Programación de aula multinivel: didáctica multigrado.

Metodologías vivas y activas.

Contenidos

La escuela abierta y conectada al mundo: posibilidades específicas de las

TIC (tecnologías de la información y la comunicación), las TAC (tecnologías del aprendizaje y el conocimiento) y las TEP (tecnologías del empoderamiento y la información).

La escuela abierta a la comunidad.

Organización de espacios y tiempos.

Recursos humanos y materiales: el territorio local y la comunidad.

El papel de la evaluación cualitativa y formativa.

Reconocer la diversidad en la práctica educativa en contextos rurales; planificar el proceso de enseñanza-aprendizaje en centros educativos ubica-

Competencias especí- dos en contextos rurales a partir de la innovación y la reflexión integrada.

ficas

Analizar e interpretar modelos de innovación e investigación (etnográfica)

aplicados a la educación en el territorio rural.

Diseñar un modelo de escuela rural en la sociedad digital.

Fuente: elaboración propia.

Por otra parte, la Universidad de Zaragoza lleva ya algunos cursos ofertando el estudio propio previamente denominado Máster Propio en Educación y Desarrollo Rural. Y en el curso 2019-2020 se oferta la segunda edición bajo la modalidad online de Experto Universitario en Educación en Territorios Rurales de la Facultad de Ciencias Sociales y Humanas de Teruel. Dicha modalidad facilita la realización de formación en ER a un amplio abanico de personas por ser no presencial y permite realizarlo con mayor autonomía. Las finalidades de este son formar profesionales que sean capaces de ejercer como docentes valorando la realidad que los rodee en cada momento, que participen de forma activa e implementen proyectos que conlleven la participación de toda la comunidad rural a la vez que, mediante las intervenciones pedagógicas, se promueve la identidad rural y los valores propios (Universidad de Zaragoza, 2019). Para todo esto, es necesaria una excelente coordinación de distintos sectores e instituciones, que a su vez promuevan la investigación en temas relacionados con ER y educación, junto con las metodologías que se utilizan en estos centros rurales.

Algunas facultades, cada vez más, incluyen en sus programaciones la ER (Chaparro \& Santos, 2018b). Un claro ejemplo de ello es la Facultad de Ciencias Humanas y de la Educación de Huesca, a quien en el curso 2019-2020 se le ha concedido un Programa de Innovación Estratégica de Titulaciones titulado «La profesión docente en el ámbito rural: formación para futuros maestros desde las 
vivencias de maestros con gran trayectoria en ER» (PIET_19_422), en el que se encuentra enmarcada esta investigación.

\section{Método}

Esta investigación responde a un enfoque cuantitativo bajo un diseño descriptivo de encuesta y de carácter no experimental basado en un cuestionario. Este tipo de estudios pretenden describir la frecuencia y las características más importantes de una realidad o fenómeno.

\subsection{Objetivos}

Los objetivos que se plantean para la presente investigación son analizar y profundizar en las valoraciones y percepciones de los estudiantes en lo referente a su formación inicial en ER, la posible influencia del contacto previo con zonas y/o centros rurales en las mismas y visibilizar algunas posibles alternativas y propuestas de mejora para la formación en dicha materia.

\subsection{Población y muestra}

La muestra de este estudio ha estado constituida por estudiantes universitarios que, actualmente, se encuentran matriculados en tercer y cuarto curso del Grado de Magisterio en Educación Infantil y en Educación Primaria en la Facultad de Ciencias Humanas y de la Educación de Huesca. El tipo de muestreo fue aleatorio simple, lo que supone que los datos obtenidos, en términos de representatividad muestral, se refieren al conjunto de la muestra y, en consecuencia, a la población diana. Se consideró como población a todas y todos los estudiantes matriculados en los últimos cursos de ambos Grados - lo que asciende a 595 sujetos-, pues ellos son los que han superado el ecuador de la formación universitaria cursando así un mayor número de asignaturas del Grado. Finalmente, la muestra quedó conformada por 113 sujetos de ambos sexos. El margen de error de la muestra es del $8,3 \%$ y el nivel de confianza de esta del $95 \%$. En la siguiente tabla (Tabla 5) se describe con mayor profundidad la muestra.

Tabla 5.

Descripción de la muestra: estadísticos descriptivos

\begin{tabular}{lllllll}
\hline & Género & $\begin{array}{l}\text { Curso de } \\
\text { Grado }\end{array}$ & Grado & $\begin{array}{l}\text { Vivir } \\
\text { zona rural }\end{array}$ & $\begin{array}{l}\text { Piensan tra- } \\
\text { bajar en es- } \\
\text { cuela rural }\end{array}$ & $\begin{array}{l}\text { Quieren } \\
\text { trabajar en } \\
\text { escuela ru- } \\
\text { ral }\end{array}$ \\
\hline Estudiantes & $6,3 \%$ & $28 \%$ & $77,5 \%$ & $55 \%$ & $31,5 \%$ & $26,1 \%$ \\
del Grado de & Hombres & $3^{\circ}$ curso & Infantil & No & No & No \\
Magisterio & $93,7 \%$ & $72 \%$ & $22,5 \%$ & $45 \%$ & $68,5 \%$ & $73,9 \%$ \\
& Mujeres & $4^{\circ}$ curso & Primaria & Sí & Sí & Sí \\
\hline
\end{tabular}

Fuente: elaboración propia

\subsection{Instrumento}

El instrumento utilizado para llevar a cabo el presente estudio ha sido un cuestionario ad-hoc basado en Ruiz y Ruiz (2017) y Del Valle, Díaz, Pérez y Vergara (2018). Se trata de un cuestionario compuesto por 19 ítems, todos ellos con respuesta de escala Likert de 1 a 5 - siendo 1 muy en desacuerdo y 5 muy de acuerdo- . A su vez, incluye una primera parte que recoge los datos sociodemográficos. Con el propósito de clarificar los conceptos objeto de estudio, se procede a especificar las dimensiones que componen el instrumento utilizado, que son: formación universitaria, creencias de sí mismo y perspectivas de la formación y sugerencias.

Con la finalidad de mejorar el cuestionario, se han controlado posibles sesgos que afectarían a su validez y fiabilidad. Además de que los cuestionarios en los que se basa el presente estudio están previamente validados, se sometió al nuevo cuestionario creado (por ser seleccionadas preguntas de ambos) a una valoración de juicio de expertos, contando con 10 jueces expertos que analizaban la congruencia y claridad de los ítems para los estudiantes universitarios. También se tuvo en cuenta el 
Alfa de Cronbach de cada una de las dimensiones del cuestionario. En la dimensión 1 «formación universitaria» se obtuvo 0,76; en la dimensión 2 «creencias de sí mismo», un 0,89 y en la última dimensión el Alfa de Cronbach es 0,88; todas ellas superan el 0,7 por lo que se considera un instrumento fiable.

\subsection{Procedimiento de recogida y análisis de datos}

Para la obtención de los datos, el cuestionario fue enviado por correo electrónico a la población diana. Una vez recibidos los mismos, se codificaron las respuestas y los datos fueron analizados mediante el paquete estadístico SPSS versión 24, con el que se trabajaron los estadísticos descriptivos, medias, desviaciones típicas, frecuencias, correlaciones, etc.

\section{Resultados}

Los resultados derivados del presente estudio se han estructurado en torno a las tres dimensiones de análisis: formación universitaria, creencias de sí mismo, perspectivas y sugerencias. Y se han plasmado gráficamente, de forma que se facilite así su comprensión.

El análisis descriptivo de las distintas dimensiones que conforman el cuestionario nos indica que la primera dimensión denominada «formación universitaria» tiene puntuaciones bajas, lo que implica que la formación universitaria recibida sobre ER no es bien valorada. Tanto es así, que los universitarios manifiestan que no existe ninguna asignatura específica de ER y tan solo se trabajan algunos contenidos en algunas asignaturas del Grado. En cuanto a la segunda dimensión «creencias de sí mismo», las puntuaciones medias son ajustadas, todas ellas en torno a 2,5 puntos (sobre un máximo de 5). Estas puntuaciones muestran que las creencias sobre sí mismos en cuanto a sus habilidades, capacidades y competencias y la preparación para afrontar su profesión en la ER, no están claras y las opiniones son dispares. Entre ellas existe mayor cantidad de valoraciones bajas, aunque cabe destacar que la valoración más elevada es la que hace referencia a su percepción sobre su capacidad y competencia para trabajar en la ER. En relación con la última dimensión "perspectivas de la formación y sugerencias», se señalan las altas puntuaciones en las medias, lo que supone que los estudiantes se muestran de acuerdo y muy de acuerdo con la gran mayoría de los ítems de este bloque. Manifiestan la necesidad de buscar soluciones para poder así recibir mayor formación sobre ER, como demuestran puntuando positivamente cualquiera de las sugerencias que se les plantean en el cuestionario (charlas, prácticas, visitas a ER, etc.). Las de mayor valoración son la visita a una ER, clases prácticas en las que se diferencie la ER de la urbana, mayor difusión de información sobre ER y realizar un periodo de prácticas escolares en una ER. Por el contrario, la peor puntuación hace referencia a la formación teórica en ER, por lo que los estudiantes prefieren, evidentemente, formarse en la didáctica y desde un enfoque práctico.

Tabla 6.

Estadísticos descriptivos

M DT

Formación universitaria (Rango 1-5)

1. Durante mis años de Grado he sido formado en escuela rural.

$2,38 \quad 1,000$

2. Considero que he recibido la suficiente formación en escuela rural du-

2,12 $\quad 988$ rante el grado de magisterio.

3. Durante el Grado la formación recibida sobre escuela rural formaba parte de una asignatura.

4. Durante el Grado la formación recibida sobre la escuela rural era una $\quad 1,51 \quad, 872$ asignatura específica sobre la escuela rural.

5. Durante el Grado he recibido una formación inicial adaptada a las ne- $\quad 2,16 \quad 1,066$ cesidades didácticas y organizativas del medio rural.

Creencias de sí mismo (Rango 1-5)

6. Siento que tengo suficientes habilidades para abordar la enseñanza en 2,51 la escuela rural, con alumnado de distintas edades. 


\begin{tabular}{llc}
\hline 7. $\begin{array}{l}\text { Tengo la convicción de que puedo afrontar cualquier situación en una } \\
\text { escuela rural. }\end{array}$ & 2,67 &, 994 \\
8. $\begin{array}{l}\text { Creo que soy una persona bastante capacitada y competente para tra- } \\
\text { bajar en una escuela rural. }\end{array}$ & 3,13 &, 992 \\
9. $\begin{array}{l}\text { Creo que estoy preparado(a) y bastante capacitado(a) para desenvol- } \\
\text { verme correctamente como maestro en una escuela rural. }\end{array}$ & 2,80 & 1,007 \\
\hline
\end{tabular}

Perspectivas de la formación y sugerencias (Rango 1-5)

10. Para ser maestro/a de escuela rural, considero importante hacer algún periodo de prácticas escolares del Grado en escuelas ubicadas en este medio.

11. Me supondría algunos inconvenientes que me asignasen trabajar en una escuela rural.

12. Creo que es necesaria una formación teórica extra sobre estos centros rurales.

13. Vería conveniente que se impartiera en la Universidad alguna clase práctica de escuela rural para ver las diferencias respecto a las escuelas urbanas.

14. Sugeriría a la Universidad que programase charlas de profesionales sobre cómo se trabaja en estas ER.

15. Sugeriría que la Universidad facilite más información sobre las ER en el Grado.

16. Sugeriría la posibilidad de hacer un período de prácticas en escuela rural.

4,42 $\quad, 837$

$2,53 \quad 1,256$

$4,10 \quad 1,009$

4,55 $\quad, 772$

4,32 945

4,5 $\quad, 785$

4,33 908

4,20 923

17. Sugeriría realizar trabajos relacionados con las ER en alguna asignatura que contemple una parte teórica sobre estos centros.

18. Sugeriría visitar alguna escuela rural para conocer su funcionamiento de forma directa.

19. Me interesa formarme en escuela rural para poder dar las respuestas adecuadas a los alumnos y a los agentes implicados en el entorno rural de esa escuela.

Fuente: elaboración propia.

Las correlaciones establecidas entre las distintas variables (Tabla 7) indican que cuanto mejor valorada está la formación universitaria, mayores capacidades perciben los estudiantes que tienen para trabajar en la ER. Sin embargo, a mayor puntuación en la variable formación universitaria no existe relación con las variables de perspectivas de la formación y sugerencias. Esto es, los alumnos que consideran más adecuada la formación creen tener buenas habilidades para ejercer su profesión de maestro/a en la ER y, por lo tanto, no realizan sugerencias destacadas en torno a su formación, lo que se refleja en puntuaciones menores que el resto en las propuestas.

Tabla 7.

Correlaciones entre las variables evaluadas

123

1. Formación universitaria

2. Creencias de sí mismo 0,25**

3. Perspectivas de la formación y sugerencias $\quad-0,10 \quad 0,01$

Nota. $* * p<0,01$

Los participantes de la investigación que a lo largo de su vida han estado en contacto con el medio rural y/o han estudiado en una ER se sienten más capacitados para trabajar como maestros y maestras en estos centros rurales que aquellas personas que no han estudiado en ellos. Por otra parte, destacar 
que todos, es decir, tanto los que han estudiado en una ER como los que no, están concienciados de la necesidad de formación en ER que deben recibir (Tablas 8 y 9).

Tabla 8.

Comparaciones en variables evaluadas según «He estudiado en una escuela rural»

\begin{tabular}{llll}
\hline & $\begin{array}{l}\text { He estudiado en escuela } \\
\text { rural }\end{array}$ & $\begin{array}{l}\text { No he estudiado en es- } \\
\text { cuela rural }\end{array}$ & \\
\hline & M(DT) & M(DT) & F \\
\hline Formación universitaria & $11,32(4,02)$ & $10,76(3,70)$ &, 396 \\
Creencias de sí mismo & $12,65(3,02)$ & $10,34(3,50)$ & 2,356 \\
$\begin{array}{l}\text { Perspectivas de la formación y } \\
\text { sugerencias }\end{array}$ & $44,57(4,97)$ & $43,04(6,50)$ & 2,062 \\
\hline
\end{tabular}

Fuente: elaboración propia.

Tabla 9.

Comparaciones en variables evaluadas según «He vivido o vivo actualmente en zona rural»

\begin{tabular}{llll}
\hline & $\begin{array}{l}\text { Vivo/He vivido en zona } \\
\text { rural }\end{array}$ & $\begin{array}{l}\text { No he vivido en zona } \\
\text { rural }\end{array}$ & \\
\hline & M(DT) & M(DT) & F \\
\hline Formación universitaria & $11,00(4,09)$ & $10,90(3,59)$ &, 81 \\
Creencias de sí mismo & $12,36(2,99)$ & $10,08(3,597)$ & 2,53 \\
$\begin{array}{l}\text { Perspectivas de la formación y su- } \\
\text { gerencias }\end{array}$ & $44,66(5,34)$ & $42,64(6,50)$ & 3,93 \\
\hline
\end{tabular}

Fuente: elaboración propia.

Tabla 10.

Comparaciones en variables evaluadas según «Tengo pensado trabajar en una escuela rural»

\begin{tabular}{llll}
\hline & $\begin{array}{l}\text { Tengo pensado trabajar en } \\
\text { escuela rural }\end{array}$ & $\begin{array}{l}\text { No tengo pensado trabajar } \\
\text { en escuela rural }\end{array}$ & \\
\hline & M(DT) & M(DT) & F \\
\hline Formación universitaria & $10,69(3,54)$ & $11,49(4,32)$ & 2,80 \\
Creencias de sí mismo & $11,30(3,40)$ & $10,69(3,77)$ &, 05 \\
$\begin{array}{l}\text { Perspectivas de la forma- } \\
\text { ción y sugerencias }\end{array}$ & $44,47(4,82)$ & $41,54(7,84)$ & 12,20 \\
\hline
\end{tabular}

Fuente: elaboración propia.

Tabla 11

Comparaciones en variables evaluadas segin "Quiero trabajar en una escuela rural》

\begin{tabular}{llll}
\hline & $\begin{array}{l}\text { Quiero trabajar en es- } \\
\text { cuela rural }\end{array}$ & $\begin{array}{l}\text { No quiero trabajar en es- } \\
\text { cuela rural }\end{array}$ & \\
\hline & M(DT) & M(DT) & F \\
\hline Formación universitaria & $10,76(3,44)$ & $11,48(4,72)$ & 7,79 \\
Creencias de sí mismo & $11,24(3,37)$ & $10,72(3,93)$ & 1,54 \\
$\begin{array}{l}\text { Perspectivas de la formación y } \\
\text { sugerencias }\end{array}$ & $44,40(5,29)$ & $41,14(7,42)$ & 7,54 \\
\hline Fuente: elaboración propia. & & &
\end{tabular}

Fuente: elaboración propia. 
En vista de los distintos resultados obtenidos, se podría concluir que el haber tenido un contacto con la zona rural y, por supuesto, haber estudiado en una ER parece influir en la valoración de las capacidades, habilidades de sí mismo, así como en los recursos para afrontar situaciones en estos entornos rurales. Pero lo que los estudiantes del Grado de Magisterio trasladan de manera explícita es que se necesita mayor formación en ER para poder desarrollar su profesión adecuadamente y así poder dar respuesta a la especificidad de esa escuela.

\section{Discusión y conclusiones}

Los actuales estudiantes del Grado de Magisterio van a ser los maestros de generaciones próximas de niños y niñas, bien sea en colegios públicos o privados, en zonas urbanas o en zonas rurales. Evidentemente, la profesión es la misma, ser maestro o maestra, y depende de muchos factores, entre ellos el contexto, pero de lo que no debería depender nunca es de su formación.

Tal y como se ha manifestado a lo largo de este estudio, la ER es una institución con características peculiares, con un potencial educativo incuestionable y con una identidad propia que lógicamente la hace diferente a la escuela urbana (Hamodi \& Aragués, 2014). Coincidimos con Chaparro y Santos (2018a) en que trabajar en escuelas incompletas, aula multigrado, colegios rurales agrupados... que acogen a grupos naturales y pocos numerosos, donde destaca la diversidad cronológica, diferentes ritmos de aprendizaje, diferentes niveles madurativos y la atención individualizada al alumnado de distintos grados, nada tiene que ver con el trabajo que se desarrolla en las escuelas urbanas completas y con grupos cronológicamente homogéneos. Y desde los Grados de Magisterio se debería responder a esta realidad ofertando asignaturas muy variadas, y que a su vez engloben todos los contextos, tanto urbanos como rurales, en los que los futuros maestros realizarán su labor, para poder así eliminar la discrepancia existente entre la preparación de los maestros y la realidad del contexto educativo (Dlamini, 2018). Porque indudablemente los futuros maestros deben ser formados para cualquier destino y situación que puedan experimentar.

Sin embargo, en el presente, los futuros maestros manifiestan que la formación universitaria en escuela rural no es suficiente, es muy transversal, de corte muy teórico y poco ligada a la realidad práctica. Estos resultados refrendan lo que numerosos estudios han evidenciado, que la formación de maestros para el desarrollo de competencias en ER resulta bastante reducida (Boix, 2004; Bustos, 2008, 2012; Chaparro \& Santos, 2018a; Hamodi \& Aragués, 2014; Hinojo, Raso, \& Angustias, 2010; Jiménez, 2009).

Asimismo, manifiestan que tener arraigo con zonas rurales influye en la percepción de sus capacidades y competencias. Es decir, los futuros maestros que tienen contacto con las zonas rurales se consideran capacitados y con suficientes habilidades para desarrollar adecuadamente su profesión de maestro y maestra en las ER, lo que no ocurre con el resto de los compañeros ajenos al medio rural. Estas experiencias previas en los contextos rurales o incluso en las ER lógicamente pueden influir en sus perspectivas de trabajo, pero no deben ser determinantes para filtrar si consideran que tienen suficientes estrategias para desempeñar su profesión de maestro o no. Por lo tanto, para evitar estas diferencias se debería reflexionar sobre ello e intentar modificar la formación desde la base, es decir, la formación inicial de los maestros. Porque los maestros necesitan tener recursos para atender las peculiaridades y especificidades del ámbito rural, es decir, precisan «una formación diferente para enseñar en la escuela rural» (Abós, 2007, p. 86).

De esta forma, incorporando la formación específica en escuela rural que los maestros demandan en el presente estudio e incluso teniendo en cuenta sus sugerencias como posibilidades de mejora, se satisfarían sus necesidades y además se les proporcionaría una formación integral que les permitiría ejercer magníficamente como maestros y maestras de Educación Infantil o Educación Primaria.

Estas circunstancias se deberían afrontar, no únicamente desde la universidad, sino en colaboración con otras instituciones o centros que tal vez deberían aportar su esfuerzo para mejorar la formación docente. Pues la formación de un maestro no finaliza con el grado universitario, previsiblemente seguirán formándose, pero la propia formación permanente, como hemos podido valorar, no les está ofreciendo actualmente alternativas, este es otro eslabón de la cadena sobre el que se debería trabajar. Definir programas formativos coordinados que permitan la formación continua, la actualización para poder responder a contextos educativos rurales cuyos rasgos definitorios vienen condicionados por la diversidad en el sentido más amplio de la palabra. 
Por todo ello, la formación específica en ER debe dejar de ser una tarea pendiente como lo ha sido hasta el momento y una demanda histórica (Cragnolino \& Lorenzatti, 2015). Con esta finalidad, en los estudios universitarios deben ofrecerse las suficientes estrategias como para lograr la equidad en la calidad de las ER (Azano \& Stewart, 2015), para apoyar a otros investigadores que se sienten incapaces de determinar qué características personales son las más adecuadas para ser un gran maestro o maestra en las ER (Hudson \& Hudson, 2008). Así pues, cualquier persona que reciba una buena formación, independientemente de que hayan estudiado en una ER o vivido en una zona rural, se desenvolverán con igual éxito en las ER, en contraposición a lo que algunos investigadores insinuaban (Hudson \& Hudson, 2008).

En definitiva, la presente investigación permite un acercamiento al análisis que realizan los futuros maestros egresados sobre su formación inicial en ER y la valoración que hacen de sus competencias en este ámbito. La limitación con la que esencialmente nos topamos es la dificultad para generalizar los resultados. Por ello, entendemos que no podemos quedarnos aquí y como prospectiva de investigación, queda como tarea pendiente realizar un análisis en profundidad de la formación inicial impartida por todas y cada una de las universidades con el objeto de ajustar los planes de formación de maestros a la realidad de nuestro país y responder a la ruralidad de este, porque solo a través de la investigación se podrá avanzar hacia la mejora. Así se logrará que sea una idea del pasado el que la formación del profesorado novel en el ámbito rural es más bien escasa o inexistente (Azano \& Stewart, 2015).

\section{Referencias}

Abós, P. \& Boix, R. (2017). Evaluación de los aprendizajes en escuelas rurales multigrado. Aula abierta, 45, pp. 41-48. doi: 10.17811/rifie.45.2017.41-48.

Avery, L. M. (2013). Rural Science Education: Valuing Local Knowledge. Theory into Practice, 52(1), pp. 28-35. doi: 10.1080/07351690.2013.743769.

Azano, A. P., \& Stewart, T. T. (2015). Exploring Place and Practicing Justice: Preparing Pre-Service Teachers for Success in Rural Schools. Journal of Research in Rural Education, 30(9), pp. 1-12.

Berlanga, S. (2014). Presente y futuro de la Escuela Rural. Fórum Aragón, 11, pp. 1-16.

Boix, R. (2004). La escuela rural: funcionamiento y necesidades. Madrid: Cisspraxis.

Boix, R. \& Bustos, A. (2014). La enseñanza en las aulas multigrado: Una aproximación a las actividades escolares y los recursos didácticos desde la perspectiva del profesorado. Revista Iberoamericana de Evaluación Educativa, 7(3), pp. 29-43.

Bustos, A. (2008). Docentes de Escuela Rural. Análisis de su formación y sus actitudes a través de un estudio cuantitativo en Andalucía. Revista de investigación educativa, 2(26), pp. 485-519.

Bustos, A. (2012). La escuela rural. Granada: Octaedro.

Catálogo de actividades promovidas por la administración educativa 2018-2019. Consultado el 29 de diciembre de 2019. Recuperado de https://formacionprofesorado.aragon.es/formacion-inicialnovel-y-permanente/catalogo-de-acciones-formativas-curso-18-19/.

Chaparro, F. \& Santos, M. L. (2018a). La formación del profesorado para la Escuela Rural: una mirada desde la educación física. Revista Electrónica Interuniversitaria de Formación del Profesorado, 21(3), pp. 93-107. doi: doi.org/10.6018/reifop.21.3.321331.

Chaparro, F. \& Santos, M. L. (2018b). Teaching competences for the rural school in the initial training. Analysis of results of a multiple study. ESHPA - Education, Sport, Health and Physical Activity. 2(2), pp. 177-191. doi: http://hdl.handle.net/10481/51755.

Cragnolino, E. \& Lorenzatti, M. C. (2015). La formación docente rural en Uruguay. En M. C. Lorenzatti \& V. Ligorria (Eds.), Educación de Jóvenes y Adultos y Educación Rural: Aportes para la formación de futuros maestros (pp. 98-107). Argentina: UniRío Editora.

Cifuentes, J. E. (2015). Una mirada a la escuela desde las voces de unos estudiantes del sector rural. Revista Virtual Universidad Católica del Norte, 45, pp. 159-172.

Dlamini, M. E. (2018). Preparing student teachers for teaching in rural schools using work integrated learning. The Independent Journal of Teaching and Learning, 13(1), pp. 86-95.

Decreto 83/2018, de 8 de mayo, del Gobierno de Aragón, por el que se crea el Observatorio de la Escuela Rural en Aragón, y se regula su organización y funcionamiento, Zaragoza: Boletín Oficial de Aragón (2018). 
Del Valle, M., Díaz, A., Pérez, M. V. \& Vergara, J. (2018). Análisis Factorial Confirmatorio Escala Autoeficacia Percibida en Situaciones Académicas (EAPESA) en Universitarios Chilenos. Revista Iberoamericana de Diagnóstico y Evaluación (RIDEP), 49(4), pp. 97-106. doi: doi.org/10.21865/RIDEP49.4.08.

Efe Madrid/Zaragoza (2 de octubre de 2019). La población rural cae un 4,2 \% en Aragón desde 2000, hasta los 421.000 habitantes. Recuperado de: https://www.heraldo.es/noticias/aragon/2019/10/02/la-poblacion-rural-cae-un-4-2-en-aragon-desde-2000-hasta-los-421-000-habitantes-1336675.html.

Formación académica de la Universidad de Zaragoza: oferta de estudios propios. Consultado el 27 de diciembre de 2019. Recuperado de https://academico.unizar.es/formacion-permanente/oferta-de-estudios-propios.

Gracia, M. (12 de septiembre de 2019). La vuelta al cole en la escuela rural. Aragón digital. Recuperado de: https://www.aragondigital.es/2019/09/12/la-vuelta-al-cole-en-la-escuela-rural/.

Guía docente de la asignatura Currículum en Contextos Diversos. Grado de Magisterio en Educación Primaria. Recuperado de: https://estudios.unizar.es/estudio/asignatura?anyo_academico=2019\&asignatura_id=26601\&estudio_id=20190116\&centro_id=202\&plan_id_nk=299.

Guía docente de la asignatura La Escuela como Espacio Educativo. Grado de Magisterio en Educación Primaria. Recuperado de: https://estudios.unizar.es/estudio/asignatura?anyo_academico=2019\&asignatura_id=26603\&estudio_id $=20190116 \& c e n t r o \_i d=202 \& p l a n \_i d \_n k=299$.

Guía docente de la asignatura La Escuela de la Educación Infantil. Grado de Magisterio en Educación Infantil. Universidad de Zaragoza. Recuperado de: https://sia.unizar.es/doa/consultaPublica/look[conpub]MostrarPubGuiaDocAs?entradaPublica=true\&idiomaPais=es.ES\&_anoAcademico $=2019 \& \_$codAsignatura $=26524$.

Hamodi, C. \& Aragués, S. (2014). La escuela rural: ventajas, inconvenientes y reflexiones sobre sus falsos mitos. Palobra, 14, pp. 46-61. doi: doi.org/10.32997/2346-2884-vol.14-num.14-2014-48.

Hinojo, F., Raso, F., \& Angustias, M. (2010). Análisis de la organización de la Escuela Rural en Andalucía: problemática y propuestas para un desarrollo de calidad. Reice, 8(1), pp. 79-105. Recuperado de: http://www.rinace.net/reice/numeros/arts/vol8num1/art6.pdf.

Hudson, P., \& Hudson, S. (2008). Changing Preservice Teachers' Attitudes for Teaching in Rural Schools. Australian Journal of Teacher Education, 33(4), pp. 67-77. doi: dx.doi.org/10.14221/ajte.2008v33n4.6.

Jiménez, J. (2009). La escuela rural. Revista digital: Innovación y experiencias educativas, 23, pp. 1-10.

Ley Orgánica de Mejora de la Calidad Educativa, Madrid: Boletín Oficial del Estado (2013).

Mallada, L. M. (2012). La dirección de un centro rural agrupado. Fórum Aragón, 5, pp. 10-16.

Masinire, A., Maringe, F. \& Nkambule, T. (2014). Education for rural development embedding rural dimensions in initial teacher preparation, Perspectives in education, 32(3), pp. 146-158. doi: hdl.handle.net/11660/3627.

Memoria de Verificación del Grado de Magisterio en Educación Infantil de la Universidad de Zaragoza. Recuperado de https://academico.unizar.es/ofiplan/memorias-verificadas-de-titulos-degrado-sociales-y-juridicas.

Memoria de Verificación del Grado de Magisterio en Educación Primaria de la Universidad de Zaragoza. Recuperado de https://academico.unizar.es/ofiplan/memorias-verificadas-de-titulos-degrado-sociales-y-juridicas.

Ministerio de Educación y Formación Profesional (2019). Informe 2019 sobre el sistema educativo español. Consejo Escolar del Estado. Secretaría General Técnica: Madrid.

Monge, R. (2019). Escuelas unitarias aragonesas, pequeñas pero no invisibles. Enlace, 29, pp. 28-38.

Moreno, A. (2000). La formación del maestro entre los retos de la sociedad postmoderna. Revista Electrónica Interuniversitaria de Formación del Profesorado, 1(3), pp. 1-11.

Orden ECI/3854/2007, de 27 de diciembre, por la que se establecen los requisitos para la verificación de los títulos universitarios oficiales que habiliten para el ejercicio de la profesión de Maestro en Educación Infantil, Madrid: Boletín Oficial del Estado (2007).

Orden ECI/3857/2007 de 27 de diciembre, por la que se establecen los requisitos para la verificación de los títulos universitarios oficiales que habiliten para el ejercicio de la profesión de Maestro en Educación Primaria, Madrid: Boletín Oficial del Estado (2007). 
Recio, M. (2018). La Escuelas Rurales en España. Informe CCOO: La escuela rural en el siglo XXI. https://fe.ccoo.es/8f31b190cd9da00e51ca9b1f2cf4d348000063.pdf.

Ruiz, N. \& Ruiz-Gallardo, J. R. (2017). Colegios rurales agrupados y formación universitaria. Revista de currículum y formación del profesorado, 21(4), pp. 215-240.

Vázquez, R. (2008). Las Escuelas Rurales: Un lugar en ninguna parte. Las ciudades invisibles del mundo educativo. REIFOP, 11(1), pp. 53-58. 